\title{
EDUCACIÓN COOPERATIVA: UNA
}

\section{ALTERNATIVA PARA GENERAR \\ CONOCIMIENTO SOCIAL TRANSFORMADOR}

\section{Elsi Belén Valenzuela Rotondaro}

Doctora en ciencias de la Educación (UNERG), Postdoctorado en Investigación Educativa, UPEL, Postdoctorante en Investigación y Gerencia de Proyectos Convenio RIEAC-Universidad Latinoamericana del Caribe. Investigador certificado en el Programa de Estímulo a la Investigación e Innovación (PEII) Categoría B - 2015. Docente Investigador UNERG, evalenzuela@unerg.edu.veelsivalenzuela@ gmail.com

\section{Resumen}

La praxis educativa tradicional requiere de un cambio donde los egresados universitarios sean formados con competencias emprendedoras para organizarse junto con los demás actores de su comunidad a los efectos de generar desarrollo socioeconómico en base a las necesidades detectadas bajo un esquema armónico sustentable. Así, esta investigación pretende reflexionar sobre el desempeño de los actores educativos y su intervención en el contexto socio-productivo bajo la perspectiva de la educación cooperativa para fomentar actitudes y aptitudes que permitan su integración como agentes de transformación social. Para ello el paradigma cualitativo resulta apropiado ya que propicia la reflexión introspectiva y vivencial sobre el desempeño de los actores en el contexto socio-productivo bajo la perspectiva de su actuación como agentes de transformación social a lo cual la etnometodología proporciona el camino a seguir. Los resultados de la intervención llevan a identificar las aristas ontoepistemicas que influyen en la investigación educativa orientada a la construcción del conocimiento social transformador, por lo que la educación cooperativa conciben a la universidad como una organización inteligente que pretende participar en su entorno local con aspiraciones globales y con conciencia y respeto a la complejidad sociobioecodinámica que caracteriza al humano y sus relaciones. 
Educación cooperativa: una alternativa para generar conocimiento social transformador.

Palabras claves: praxis educativa, educación cooperativa, transformación social.

\section{COOPERATIVE EDUCATION: AN ALTERNATIVE TO GENERATE TRANSFORMER SOCIAL KNOWLEDGE}

\section{Abstract}

The traditional educational praxis requires a change where university graduates are trained with entrepreneurial skills to organize together with other actors in their community in order to generate socio-economic development based on the needs detected under a sustainable harmonic scheme. Thus, this research aims to reflect on the performance of educational actors and their intervention in the socio-productive context under the perspective of cooperative education to foster attitudes and skills that allow their integration as agents of social transformation. For this, the qualitative paradigm is appropriate because it facilitates the introspective and experiential reflection on the performance of the actors in the socio-productive context under the perspective of their action as agents of social transformation, to which ethnomethodology provides the way forward. The results of the intervention lead to identify the ontoepistemic edges that influence educational research oriented to the Construction of Transformative Social Knowledge, which is why cooperative education conceives the university as an intelligent organization that intends to participate in its local environment with global aspirations And with conscience and respect to the sociobioecodynamic complexity that characterizes the human and its relations.

Keywords: educational praxis, cooperative education, social transformation.

\section{Introducción}

Pensar en el hecho educativo implica un debate sobre las razones teleológicas que determinan su accionar sobre la población. Para ello es necesario, partir del hecho que cada quien quiere vivir en un país que le brinde las mejores condiciones de manera individual y para su entorno más inmediato. El espíritu del asunto está en 
educar al colectivo para que aprenda a apropiarse del conocimiento mediante el empleo de estrategias significativas y vivenciales donde la construcción del saber aflora libremente y en correspondencia con las necesidades del sujeto a los efectos de facilitar su vinculación con el entorno social, económico, ecológico, entre otros. Por tanto, hay que vulcanizar los conocimientos: en el medio urbano ¿qué destreza se esperan que tenga una persona? ¿Serán las mismas que necesita el ciudadano en el medio rural? ¿Cuáles son las expectativas? La respuesta debe centrar su atención en convertir los centros educativos en unidades de producción de agentes de transformación social, refiriéndose a estos como a un grupo o asociación de personas capaz de reconocer y comprender la realidad que vive para intervenir en ella con acciones específicas para producir evolución mediante la gesta de impactos positivos en el desarrollo económico y social de las zonas geográficas donde se encuentran generando beneficios para el colectivo.

Así pues, las universidades deben desarrollar novedosos modelos educativos y mantenerse sensibilizadas con las necesidades de la sociedad. Por lo tanto, las funciones académicas, de investigación y extensión no han logrado fusionarse para que sus actores protagonicen acciones orientadas a un desarrollo humano integral con competencias, lo cual ha dificultado el impulso de cambios significativos en el quehacer cotidiano desde las diferentes aristas que caracterizan la convivencia en sociedad y haciendo énfasis particularmente en aquellos sectores socioproductivos menos favorecidos limitando la posibilidad de superar su situación. En correspondencia, las acciones que se emprenden desde la academia y la investigación se caracterizan por una pertinencia social limitada y en ocasiones sesgada a intereses particulares, es decir, orientadas al desarrollo individualista o sectorizado alejado de una visión amplia de los complejos ámbitos de la vida cotidiana y de las necesidades de la sociedad. Muchas veces se observan investigaciones sin relación, con aspectos inmediatos relativos a la complacencia de un mercado laboral específico y limitado, alejadas de los problemas sociales complejos como la erradicación de la pobreza, el deterioro del medio ambiente o el analfabetismo por ejemplo. Por tanto, los egresados cumplen con el rol de agente de transformación social de forma restringida, ya que no fue formado como emprendedor desde el descubrimiento de sus potencialidades, 
Educación cooperativa: una alternativa para generar conocimiento social transformador. ya que el proceso educativo se desvinculó del reconocimiento de sus necesidades en correspondencia con la realidad que vive: educación masificada y estandarizada.

Por tanto, la educación cooperativa centra su atención en enseńar a construir y reconstruir con las herramientas que el pensamiento reflexivo y la socialización entre pares proporcionan en la dinámica educativa y de investigación, entre facilitadores y aprendices. Es allí, donde se refleja el surgimiento de un nuevo paradigma educativo que elimina la brecha importante entre el "profesor poseedor de conocimientos" y el "alumno repositorio de información”, quienes ahora se ven como dos iguales que caminan juntos por el sendero del descubrimiento en el marco de una realidad que atañe a ambos. Ante la situación referida, esta investigación deberá responder las siguientes interrogantes para orientar la praxis educativa universitaria como productora de agentes promotores del cambio social desde la educación cooperativa:

1. ¿Cuál es el perfil de competencias que caracterizan a los futuros egresados del área de ingeniería de sistemas de la Universidad Nacional Experimental Rómulo Gallegos, (UNERG) que les permita alcanzar sus objetivos y realizar su misión, a la vez que se desarrolla integralmente, considerando los principios cooperativistas de educación, cooperación entre pares y responsabilidad social? ¿La formación cooperativa en su contexto permitirá el cambio paradigmático generando la oportunidad de una autentica transformación social?

2. ¿Qué actitudes y aptitudes deben ser proporcionadas y estimuladas para que los integrantes de una organización posean el entrenamiento y motivación adecuados para generar conocimiento social transformador?

\section{Desarrollo del trabajo}

La acción humana es la unidad mínima y fundamental de la realidad social y se define como un "comportamiento orientado hacia el logro de fines, metas u otros estados de cosas anticipados" (Parsons, 1951:53). Concepto que visto ontológicamente apunta al individualismo en virtud de que la motivación y acción consecuente es producto de un "yo y mis circunstancias" Ahora bien y 
considerando la necesidad humana de compartir con otros seres semejantes, podemos contrastar esta idea con la Teoría de la Acción Comunicativa de Habermas, quien propone un modelo donde la sociedad puede entenderse como dos formas simultáneas de racionalidad que están presentes: la racionalidad sustantiva del mundo de la vida que simboliza una perspectiva interna como el punto de vista de los sujetos que actúan sobre la sociedad y la racionalidad formal del sistema, que representa la perspectiva externa, como la estructura sistémica (la racionalidad técnica, burocratizada, de las instituciones).

Ante esta comparación, la realidad de la sociedad actual señala que el capitalismo como sistema ha impuesto patrones de acción orientados hacia fines concretos, donde la interactividad con las sociedades no existe y hay una perturbación evidente entre los intereses, claros y definidos, de las organizaciones de producción de bienes y servicios y "el mundo de la vida", expresado por los miembros de la sociedad en toda su diversidad social, académica, cultural y étnica. Se revela una brecha significativa entre este "mundo de la vida”, fuera del ámbito del sistema, es decir, los radios de acción no ponderados, ni encaminados a fines concretos, de la vida cotidiana. Por lo tanto, el estímulo principal está representado por las acciones humanas concretas y articuladas hacia la producción expedita de una respuesta a la racionalidad inhumana del sistema capitalista a través del continuo "diálogo intersubjetivo", que se produciría en el "mundo de la vida", buscando un fin concreto a través del entendimiento en el diálogo continuo con los demás, llegando a conclusiones por consenso. Así pues, la validación del nuevo conocimiento está fundamentada por la participación activa de la ciudadanía en su gestación y, por tanto, es útil para la acción social.

En tal sentido, la asociación de actores de la sociedad fundamentada en los principios y valores del cooperativismo puede comprenderse como una filosofía de vida, donde las actitudes son los pilares fundamentales para el diálogo y entendimiento, la construcción de nuevos saberes por intercambio que permiten la realización de un trabajo en armonía y con desempeño exitoso. La disposición, la alegría, el entusiasmo, el compartir, la constante motivación, la creatividad y la innovación, la amistad, el compañerismo, la solidaridad, el apoyo mutuo, la responsabilidad y la hermandad se 
Educación cooperativa: una alternativa para generar conocimiento social transformador. convierten en los cimientos que solidifican la estructura del conocimiento social transformador.

En correspondencia y aplicando los postulados de la filosofía de Owen (1816), la educación cooperativa exhibe como principal característica un diseño y planificación curricular por competencias basadas en la construcción de un estilo compartido de aprendizaje basado en la construcción de saberes con el justo reconocimiento de las fortalezas que son aprovechadas y de las debilidades que serán consideradas como oportunidades de mejora. Por lo tanto, el compartir de saberes obtenidos de las vivencias cotidianas facilita la integración del grupo, especialmente cuando se realizan los talleres o reuniones para la socialización. En consecuencia, surgen el entusiasmo, la aceptación y el dinamismo que favorece y estimula a la creatividad, por lo que emergen estrategias innovadoras capaces de generar planes de contingencia para situaciones inesperadas. Hay unión del grupo, no solo por un interés común, sino por afinidad entre las personas, quienes desarrollan lazos afectivos, amistad y hermandad, por lo tanto hay reconocimiento por la labor realizada que incrementa la confianza y la autoestima. Esta integración del grupo se refleja mediante una alineación en las directrices de trabajo y en el discurso que maneja cada participante, en el cual la constancia, motivación y fidelidad se demuestran cuando las actividades se realizan con alegría y entusiasmo.

Otro aspecto importante observable en este tipo de experiencias educativas, centra su atención en el estímulo constante a la participación en un ambiente de confianza y alegría es determinante en el descubrimiento y empleo de potencialidades ocultas en los individuos. En tal sentido, es propicia la selección cuidosa de estrategias que propicien el respeto y equilibrio, donde el sentido de la competencia es sustituido por la filosofía ganar-ganar, en la cual todos las personas alcanzan el conocimiento y disfrutan de experiencias en un proceso de aprendizaje continuo para el desarrollo de una nueva generación con conciencia ecológica y visión planetaria.

Esta a su vez, se convierte en agente de motivación, reflexión y planificación permanente para que la satisfacción y el amor por lo que se hace se cultiven exitosamente logrando transformación de realidades en consenso. La consecuencia inmediata de este interactuar recursivo de actitudes ventajosas es la autorrealización del 
individuo en virtud que se siente aceptado por el grupo y como agente partícipe de las acciones que se realizan, aspecto indispensable en la cohesión e integración del conjunto de individuos. Se aprecia la capacidad de aceptación de las situaciones vividas, que resulta beneficioso para el desarrollo personal y profesional del individuo ya que asume las experiencias como enseńanzas que permiten una mejor actuación en situaciones similares futuras. El factor emocional surge como abono para que la construcción del conocimiento se internalice con mayor facilidad y permanencia, propiciando la amistad y el amor entre quienes comparten el trabajo y la investigación como pilares de desarrollo integral. Cabe destacar que estos aspectos representan algo que va más allá de una filosofía de trabajo cooperativo tal y como lo planteó Owen (1816). Es, y de manera significativa, un modo de vivir y de convivir en armonía con el prójimo, en cooperación para la acción y con el beneficio de un desarrollo socioproductivo armónico, capaz de lograr la sustentabilidad, la sostenibilidad y en consecuencia un desarrollo verdaderamente endógeno.

De hecho, es necesaria la consideración y valoración que merece el desarrollo humano, base para alcanzar la evolución desde adentro. Es decir, el progreso y avance de la sociedad es el resultado del conocimiento de sus propios problemas y requerimientos que permite la producción de soluciones adecuadas, lo cual se entiende como desarrollo endógeno. Mas (2005), señala que éste puede concebirse de dos formas fundamentales: como fin y como estrategia, como fin explica que "se define como la imagen objetivo susceptible de ser construida y alcanzada por una comunidad, en un tiempo determinado y a través de la mejor forma de utilización de todos los medios existentes" y como estrategia opina que "se trata de una acción sostenida e implantada desde y por una comunidad a través del tiempo para mejorar sus condiciones de existencia en términos integrales" (p.51).

De forma complementaria, conviene considerar el punto de vista de Villarreal \& Villarreal (2003), quienes identifican el término sustentable con la finalidad última de una organización inteligente. En tal sentido, entiéndase esta como aquella que es capaz de organizarse de acuerdo con sus necesidades, flexibilizarse en su producción y adaptarse rápidamente en su logística de comercialización 
Educación cooperativa: una alternativa para generar conocimiento social transformador.

Gráfico Nro 1: Competencias para la Educación Cooperativa.

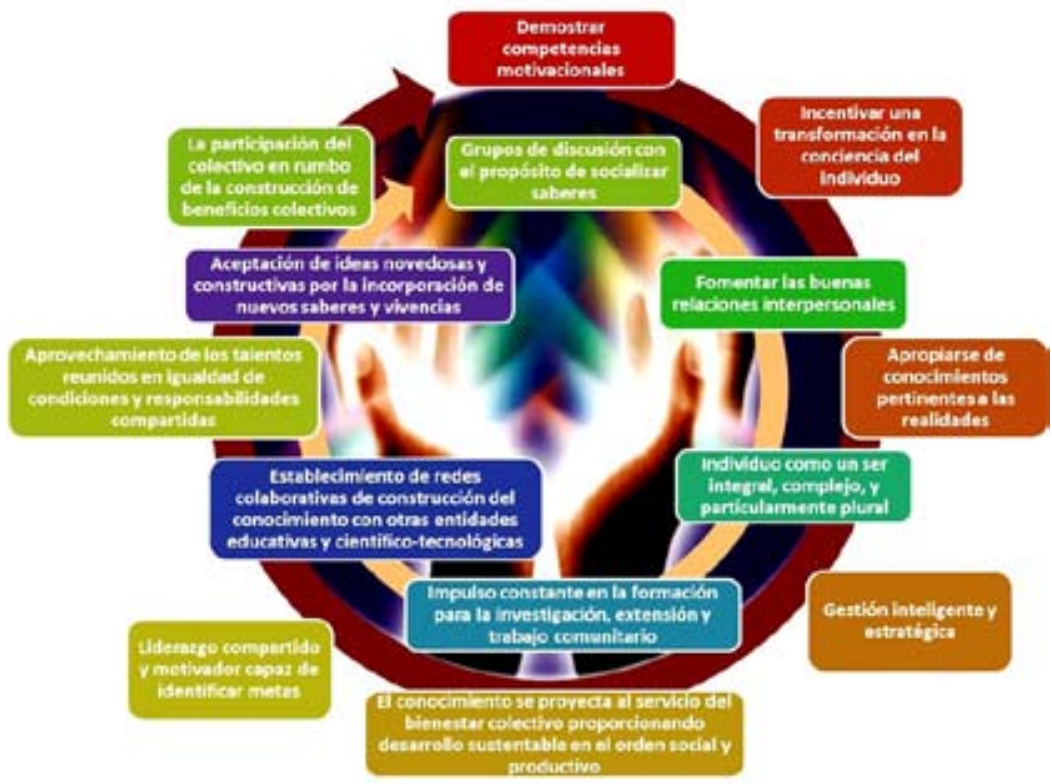

Fuente: Valenzuela (2011)

para lograr efectivamente la satisfacción de sus clientes.

Se evidencia que la capacidad de transformación total, crea y añade indefinidamente valor sobre un ente, ya sea individuos, artículos, comunidades o países, gracias al desarrollo endógeno. Ahora bien, para que éste último pueda ocurrir apropiadamente, requiere del soporte sustentable y sostenible de las acciones, que parten del hecho, que los individuos son protagonistas de la transformación social y que se reúnen para alcanzar metas comunes mediante la colaboración de saberes y recursos para la producción de medios para mejorar su calidad de vida, por lo que hemos de encontrar, que la construcción del conocimiento se hace en colectivo, dado que las vivencias son también comunes: hay un compartir evidente de conocimientos que se apropian en conjunto para ponerlos en práctica coadyuvando a la transformación de la realidad en un acto de apropiación y construcción, por lo que el acto pedagógico cobra fuerza y vigencia.

El entramado teórico que se discute consecuentemente, debe orientar sus fundamentaciones al desarrollo socioeconómico pero 
con solidez humanista. De hecho, es fundamental la integración de saberes y recursos mediante la construcción de conocimiento social transformador como unidad orientada a la producción de bienes y servicios, el cual debe garantizar su sustentabilidad. De lo contrario, se corre el riesgo inminente que el objetivo génesis no se cumpla. Acto seguido, debemos promover esfuerzos para la reflexión participativa e inteligente, donde nuestras capacidades y potencialidades estén al servicio de la investigación educativa generando alternativas que conlleven a la armonía, el respeto y la consideración de forma tal que el equilibrio entre la productividad social y económica sea efectivo y eficaz, con la calidad que todos deseamos, perdurable en el tiempo y con armonía ecológica.

Las universidades venezolanas, forman profesionales en diferentes áreas de conocimiento que buscan insertarse productivamente en el aparato socio productivo de la región a la que pertenece, sin embargo la praxis educativa, así como la investigación y la extensión que actualmente se realiza esta dista de la formación de emprendedores capaces de interactuar con su entorno bajo la figura de intelectuales orgánicos y menos aún de producir transformación social. En éste orden de ideas, la crítica que realiza la película "La Educación Prohibida" es un llamado a la reflexión sobre la praxis educativa que se lleva a cabo en nuestras escuelas y cómo podemos contribuir en una auténtica transformación en la cual se valorice al ser humano como agente participativo y protagónico del desarrollo de su entorno que debe gestar una transformación revolucionaria de la economía determinando cambios importantes en la condición socio-productiva y direccionándole hacia lo colectivo.

Consecuentemente, el aprendizaje se entiende como el proceso mediante el cual se integran conocimientos, habilidades y actitudes para conseguir cambios o mejoras de conducta. Por lo tanto, representa una acción, que toma el saber, la experiencia y la innovación como bases para generar un nuevo conocimiento de mayor pertinencia y aplicabilidad. Es un concepto que se puede aplicar a las personas y sus asociaciones razón por la cual se requieren herramientas o mecanismos que permitan convertir el conocimiento de individual en conocimiento colectivo.

Al respecto, Padrón (1994), expresa necesidades importantes e implícitas en la existencia del ser que son fundamentales y que 
Educación cooperativa: una alternativa para generar conocimiento social transformador. explican su interactuar con la sociedad. El individuo construye su propio conocimiento como resultado de las acciones vividas donde la directriz fundamental son las motivaciones, por lo que seńala: Es ya sabido que todo organismo procesa y produce información como requisito indispensable para interactuar con el medio o entorno y como base esencial para la acción: nutrición y autoconservación, protección y defensa, arraigo, expansión y otros, siempre dentro de una especial función de regulación que describe su desarrollo y subsistencia como ser vivo.

En tal sentido, se requiere comparar lo observado, vivido y sentido con los conceptos acumulados producto de la experiencia a fin de generar más experiencia. Esto es aprendizaje. Esto es construcción de la realidad, pero una realidad propia e individual, tanto como lo es el código genético ya que cada quien proporciona una interpretación particular del objeto observado o del fenómeno vivido. Por lo tanto, deben ocupar un lugar significativo, el rescate de la identidad, de los valores y principios morales culturales y éticos de los individuos y de las comunidades los cuales deben formar parte de la filosofía educativa a fin de que ésta retribuya la formación y el trabajo con el desarrollo de sus integrantes bajo un esquema de responsabilidad social. De ésta forma, el desarrollo económico sustentable, término acuñado por González (2008), asocia la utilización de recursos, del ambiente y la calidad de vida en pro de restaurar y mantener condiciones saludables que aseguren la sana existencia humana. Para el logro de esta meta se hace necesaria la identificación y conservación de los profundos valores culturales dentro de la sociedad, el cambio hacia un estilo administrativo horizontal y participativo fundamentado en un cambio paradigmático hacia el logro de la sustentabilidad.

\section{Método}

El enfoque epistémico está estructurado mediante determinados campos de observación (áreas descriptivas) y formas teóricas (áreas explicativas) que se integran para establecer preferencias o prioridades, y que, además, se cohesionan en torno a determinadas convicciones acerca de lo que conciben como conocimiento científico, con sus vías de acceso y producción del mismo y como meca- 
nismos de validación o crítica. En tal sentido, a los efectos de esta investigación se considera oportuna la adherencia a un enfoque de carácter introspectivo-vivencial. Este, se encuentra asociado con el entendimiento in situ de las conductas expresadas por los integrantes de la comunidad universitaria, partiendo de su capacidad para interactuar, reaccionar ante situaciones, aportar al desarrollo y mantenimiento de proyectos educativos con carácter socioeconómico para apalancar el desarrollo armónico sustentable.

Consecuentemente, la experiencia determina cambios en las vías y estrategias que empleamos los facilitadores para compartir los saberes, lo cual exige también la revisión de los contenidos que se abordan en los diseños curriculares en base a las exigencias de un mercado laboral dinámico, a la evolución psicosocial del individuo y a la experiencia acumulada de la labor educativa. Por lo tanto, esta experiencia queda entendida como un proceso vivencial en la que se conjugan de forma complementaria los conocimientos previos, las actitudes personales y las habilidades desarrolladas durante la cotidianidad de la praxis docente, con la intención de consolidar competencias que puedan ser transferidas a los estudiantes para el desempeño familiar, comunitario, académico y laboral quienes podrán disponer de herramientas necesarias para emprender organizaciones cooperativas que les permitan satisfacer necesidades socioeconómicas al intervenir un mundo globalizado, heterogéneo, incierto, cambiante, inestable y particularmente complejo.

El paradigma empleado centra su atención en la investigación cualitativa, en virtud de que se basa en una relación sujeto-sujeto donde se utilizan preguntas de investigación para descubrir y refinar la apreciación de la realidad donde las técnicas e instrumentos de recolección de datos se caracterizan por estar carente de mediciones numéricas como lo son las descripciones y las observaciones. Para recorrer el camino de la investigación y descubrir las nuevas luces a partir del reconocimiento interno de los actores que participan en la comunidad universitaria, es necesario establecer una metodología de trabajo que representa el vehículo mediante el cual se transita por el camino. Es así como el entendimiento, comprensión e interpretación del cómo se ejecutan y desenvuelven las actividades humanas cotidianas, en las cuales el componente individualista posee gran importancia. De hecho la condición social de los indi- 
Educación cooperativa: una alternativa para generar conocimiento social transformador.

viduos los hace interactuar con sus similares manifestando gestos y habla, entre otras muchas formas de expresión lingüística y corporal. Estos, situados dentro de un contexto particular arrojan información importante sobre los patrones conductuales, intereses, creencias, valores, actitudes y cultura de los protagonistas, cuyo significado aporta luces sobre las competencias que deben ser promovidas en el proceso de formación universitario.

En este orden de ideas, Martínez (2007), define la etnometodología, como la búsqueda empírica de los métodos empleados por los individuos para dar sentido y al mismo tiempo realizar sus acciones. Por tanto, se ha considerado como una tendencia interpretativa debido a que estudia los métodos o procedimientos con los que los integrantes de las sociedades dan sentido a la vida cotidiana o actúan en ella; desde la consideración de que el orden social está determinado por los continuos actos hermenéuticos de los sujetos implicados. Evidentemente, para iniciar el estudio la etnometodología, sugiere el empleo de "la observación independiente o participativa, según el caso, con la grabación de audio y de vídeo para poder analizar las escenas repetidas veces y, quizá, para corroborar su interpretación con una triangulación de jueces" (Martínez, 2007:124).

A los efectos de esta investigación se consideran encuentros con los informantes quienes aportaron sus consideraciones y puntos de vista para conocer e interpretar las relaciones interpersonales, los sentimientos internos y actitudes exteriorizadas en las unidades de estudio seleccionadas de forma intencional dentro del grupo de actores totales de la comunidad educativa del área de ingeniería de sistemas de la Universidad Rómulo Gallegos y su entorno, el cual estuvo conformado por 05 docentes con más de 5 años en el área, 02 egresados con más de dos años de experiencia laboral, 01 trabajador con funciones administrativas que pertenece al área desde sus orígenes y 01 trabajador con función de mantenimiento y servicio con más de 2 ańos en el área. El criterio que prevalece para su selección se basa en que los individuos son o fueron integrantes activos en el proceso de aprendizaje y descubrimiento del conocimiento, tal como lo establece el método etnográfico. Así los encuentros con las unidades de estudio seleccionadas forman parte de medio en que el investigador se desempeńa lo cual permite la captación introspectiva de la información, ya que el investigador está en la po- 
sibilidad de experimentar las mismas vivencias que los integrantes. A efectos de alcanzar la información requerida, se hace necesario reunir evidencia empírica directamente a través de un trabajo de campo, mediante el empleo de técnicas usadas con frecuencia por la etnometodología (Martínez, 2007; Colas, 1998):

a. La observación participante: en esta, la autora busca fundamentalmente el conocimiento de las personas y del lugar dónde se va a realizar la investigación, a la vez que intenta insertarse en la organización cooperativa mezclándose con las personas logrando así su aceptación como una integrante regular con el menor impacto posible.

b. Entrevistas en profundidad: consiste en un diálogo coloquial establecido entre el investigador y las unidades de estudio, Esta acción posee gran significación dado que a medida que el encuentro avanza, la estructura de la personalidad del interlocutor va tomando forma en la mente, se adquiere las impresiones de sus movimientos, audición de la voz, comunicación no verbal y a toda la amplia gama de contextos verbales.

\section{Procedimiento}

Tomando en consideración el modelo de investigación integrado propuesto por Bordeleau (2000), para el desarrollo de los recursos humanos, así como la propuesta de Martínez (2007), en cuanto al método de investigación cualitativa etnográfica, se determinan las siguientes etapas:

- Etapa 1: se debe realizar una observación del contexto a investigar con la finalidad de identificar el objeto de estudio encontrando apoyo en una revisión teórica preliminar determinando así el enunciado holopráxico (Hurtado, 2000).

- Etapa 2: se realiza una construcción del marco teórico o sintagma gnoseológico mediante la revisión de los presupuestos teóricos vigentes relacionados con las unidades de estudio: capital intelectual, cooperativismo y desarrollo sustentable.

- Etapa 3: conocido el estilo de pensamiento, el enfoque epistemológico y las opiniones surgidas de autores e investigaciones previas, se dio inicio al procedimiento de intervención de la realidad de acuerdo con los principios de la investigación cualita- 
Educación cooperativa: una alternativa para generar conocimiento social transformador. tiva etnográfica holística, entendida como la descripción cultural y su interpretación de una comunidad universitaria mediante la representación de las creencias y prácticas, que a su vez señalan cómo cada una de las partes que lo componen, contribuyen en su existencia y operatividad.

- Etapa 4: esta última parte de la categorización de las unidades de estudio y su consecuente explotación en subcategorías, las cuales permitirán extraer los patrones estructurales de las entidades observadas o informantes, y como producto de esta, la contrastación motiva una teorización capaz de interpretar las realidades encontradas y generar una conclusión razonable, confiable y validada.

- Etapa 5: para el análisis de la información obtenida se contó con el apoyo del software para análisis cualitativo de datos Atlas'ti versión 5.0, puesto que permitió fragmentar sistemáticamente los diferentes contenidos para llevarlos a categorías y códigos emergentes que combinados en una especie de juego lógico expresado por redes de interacción entre cada nivel encontrado, muestra un retrato hablado de la realidad. El abordaje de estos corpus representa el anclaje epistémico y ontológico de un conocimiento que emerge para dar respuesta a un conjunto de propósitos expresados a través de una razón teleológica donde la hermenéutica y la axiología marcan la pauta para transformar el conjunto de conocimientos individuales en colectivos.

Evidentemente los procesos mentales de clasificación, categorización, contrastación y teorización fluyen de forma recursiva en una maniobra neurológica de reflexión instantánea para permitir el surgimiento del nuevo conocimiento. En tal sentido la sistematización se hace presente para marcar una pauta de autenticidad en la interpretación y relacionamiento entre los conocimientos individuales expresados en los corpus. Para ello, resulta conveniente señalar los siguientes aspectos:

1. Los sujetos significantes: fueron abordados están distribuidos en dos momentos y a su vez cada uno fue identificado en función al rol que desempeñan en la institución a la que pertenece con la intención de respetar su privacidad.

2. Transcripción de las entrevistas: obteniendo así los "corpus" que a su vez se cargaron como documentos primarios dentro de 
una Unidad Hermenéutica. Además el sujeto significante es identificado con un seudónimo a los efectos de conservar su privacidad según se relacionó previamente.

3. Citas asociadas a las categorías preliminares: los corpus transformados en documentos primarios se fragmentaron según las categorías que se derivan de los propósitos de la investigación para identificar los puntos de encuentro entre los diferentes sujetos claves. A través de esta clasificación inicial se logra la comprensión integral del discurso por lo que emergen citas que se relaciona entre sí. Estas son señaladas mediante una numeración que permite ubicarla dentro de la totalidad del corpus. Se realiza entonces un análisis preliminar del discurso donde se visualizan las opiniones de cada informante y que permite una contrastación inicial.

4. Matriz de significados de la dimensión: las citas asociadas cada categoría se somete nuevamente a fragmentación cuyo producto genera una matriz donde emergen códigos que permite revelar significados para el sujeto que me acompaña en el recorrido y que representa el trasfondo de sus palabras. Es aquí donde el conocimiento individual comienza a relacionarse con sus pares para transformarse en colectivo proporcionando la oportunidad de generar un nuevo conocimiento sobre la relación existente entre la praxis educativa, la transformación social y el desarrollo sustentable.

5. La interpretación de los códigos emergentes: aflora de mi mano a fin de dar sentido a las relaciones develadas y desarrollando creativamente nuevas relaciones que fundamentan una aproximación teórica para la formación de emprendedores cooperativistas en el contexto de la Universidad Rómulo Gallegos bajo la perspectiva de su actuación en el aparato productivo como intelectuales orgánicos.

\section{Resultados}

Al realizar el análisis del discurso se visualizan las opiniones de cada informante el cual permite una contrastación de opiniones y en consecuencia surgen categorías que responden a los propósitos. La praxis organizacional educativa se relaciona con el proceso educativo otorgándole características de cooperatividad en la construcción del conocimiento en una relación simbiótica en- 
Educación cooperativa: una alternativa para generar conocimiento social transformador. tre los actores que participan y en la cual la complementariedad y multidisciplinariedad son factores determinantes. Ahora bien, la comprensión del desempeńo de los actores socioeducativos exige la revisión de la categoría educación cooperativa, de la cual se desprende la oportunidad explicita para la participación activa de los sujetos involucrados en la apropiación de saberes específicos que le permiten descubrir soluciones a sus interrogantes cognoscitivas. En tal sentido, La gerencia cooperativa debe ser de alta calidad técnica, pero al mismo tiempo debe ser una gerencia imbuida de los principios y valores cooperativos para que desde ella se estimule la participación de los Cooperativistas a lo largo de toda la vida de la empresa y para que dicha gerencia realice un trabajo educativo y formativo coordinado con el comité de educación cooperativa, cuya creación es obligatoria legalmente.

Es decir, el colectivo exige y dirige el viaje al descubrimiento y aprehensión de los conocimientos para alcanzar el efectivo desempeño laboral requerido para la construcción de los saberes que les guiaran al establecimiento de soluciones organizacionales que mejoren su calidad de vida, entendiendo esta como un eje transversal de la educación. Esta situación implica que dentro de las competencias que desarrolla la educación cooperativa esta la capacidad para liderar y motivar al grupo mediante actividades que estimulen el descubrimiento de las potencialidades innatas del sujeto y la superación de la timidez que envuelve su desempeño y que lo hace desarrollarse con la fortaleza que proporciona el conocimiento. Por lo tanto los contenidos programáticos en la educación cooperativa debe deben flexibilizados y ajustados a las necesidades del sujeto tal y como lo seńala el informante Caracas cuando expresa:

“..... Hay proceso de recambio en el sujeto. Tú puedes proponer un conjunto de contenidos temáticos, pero si las personas no terminan asumiéndolos como algo importante para el desarrollo de su actividad y no tienen unas perspectivas en esto, poco puede calar. No se trata sólo de la actividad pedagógica de encerrar a un conjunto de personas en un espacio y echarle la carreta de lo que sea. Las personas tienen que apropiarse de eso, que hay alguna utilidad en este conocimiento......Te digo como al principio, todo el conocimiento que pueda compartir ahí o que se pretenda construir debe ser significativo para las personas...” 
(Valenzuela, 2011 UH1-26:26)

Lo cual implica, que la transformación del sujeto individualista al ciudadano cooperativo es un proceso que ocurre a largo plazo, donde el cambio paradigmático de generación a generación se hace presente reclamando los espacios protagónicos de la existencia como seres planetarios, es decir que está relacionado con un sistema que no es lineal, sino que es de manera circular: lo que tú haces tiene relación con otros individuos sean animales, plantas, del mismo planeta y esto a su vez repercute sobre cada ser, lo cual de alguna manera se está diciendo que él tiene que cooperar en pro de esa subsistencia de ese propio planeta.

Esto es determinante para el éxito de las organizaciones que surjan como alternativas de desempeño de la investigación y de la extensión universitaria para así engranar con egresados emprendedores, lo cual es la base del desarrollo endógeno. Sin embargo, la sustentabilidad de estas empresas radica en la posibilidad de apropiarse efectivamente de los principios y valores cooperativistas dentro de una práctica educativa y de investigación desarrollada con esos mismos elementos y enfocada a solventar requerimientos humanos, tecnológicos, sociales y ambientales mediante proyectos de innovación y socio-productivos formulados con el conocimiento construido colectivamente por los actores involucrados en la realidad que se aborda. Es un proceso recursivo donde la universidad, la comunidad y el conjunto de circunstancias se funden para que emerja una posibilidad de desarrollo endógeno con propiedades sustentables y sostenibles, conformando una triada integrada por el estudiante por supuesto, que es el factor al cual se impulsa para que se abra hacia el mundo desde el reconocimiento de su realidad, luego el trabajo del docente como guía y facilitador del recorrido que expone el aprendizaje significativo y fundamentalmente, el trabajo del contexto o de la sociedad como demandante de transformación positiva con el aporte de la innovación y la puesta en práctica de los resultados de investigación pertinente, cooperativa y simbiótica.

Por lo tanto, los facilitadores deben entrar en contacto con la realidad que viven los estudiantes, y la universidad debe permitir la consolidación de esta relación propiciando los elementos académicos, logísticos y administrativos para ello. Quizás el sistema de in- 
Educación cooperativa: una alternativa para generar conocimiento social transformador. vestigación y academia se ha abocado a “ $\overline{\text { dar clases" olvidando que }}$ para compartir un conocimiento, la pertinencia y actualización del mismo son fundamentales, y la forma de conseguir tales atributos en el saber es rompiendo las barreras de las cuatro paredes del aula de clases mediante la investigación y la extensión. Es necesario por tanto, que los facilitadores y estudiantes rompan los límites físicos del aula y salgan a estudiar la realidad de las comunidades con la intención de reconocer e identificarse con la diversidad de problemas y saberes que reinan para que de forma cooperativa se construya un conocimiento valido y efectivo a la necesidad del saber. Entonces, a la luz del discurso, en esa triada, el papel de la sociedad es determinante, puesto que los conocimientos que la universidad desarrolla son para la misma sociedad quien al apropiarse de estos exige el adelanto de nuevos saberes creándose un circulo magnifico e infinito de producción intelectual.

Es decir, debe existir un diseño curricular donde están imbricados los ejes transversales relacionados con los valores y principios cooperativos y los contenidos específicos que las unidades curriculares deben proporcionar, sin abandonar elementos que permitan el surgimiento de nuevos caminos para el transitar del saber cómo lo es la función investigativa. Así las competencias desarrolladas abarcan los cinco aspectos fundamentales: básicas, para la vida, de la unidad curricular, profesional y laboral. Es por esto, que la simbiosis entre los conocimientos previos, los conocimientos que se construyen en la vida cotidiana de forma colectiva en base a los requerimientos que el entorno determina, razón por la cual las competencias adquiridas pierden vigencia porque el proceso de socialización y práctica de lo aprendido permite develar la aprehensión de competencias emergentes y novedosas que renuevan el conocimiento y lo hacen pertinente según la dinámica cambiante que exhibe la realidad compleja. El producto que surge es un individuo dinámico, proactivo y con capacidad para responder a las exigencias de su realidad, intervenirla y aportar elementos tangibles para transformarla.

En este orden de ideas, y de acuerdo con lo establecido por Parsons (1951), la acción humana es la unidad mínima y fundamental de la realidad social y se define como un "comportamiento orientado hacia el logro de fines, metas u otros estados de cosas 
anticipados" (p.53). En tal sentido la educación cooperativa puede entenderse como una filosofía de vida donde valores y actitudes son los pilares fundamentales para la realización del trabajo en armonía y con desempeño exitoso capaz de desarrollar continuamente competencias para la vida cotidiana. La disposición al trabajo, la alegría, el entusiasmo, el compartir, la constante motivación, la creatividad y la innovación, la amistad, el compañerismo, la solidaridad, el apoyo mutuo, la responsabilidad y la hermandad se convierten en los cimientos que solidifican la estructura del aprendizaje colaborativo. Entonces por qué dejar de lado a los estudiantes a la hora de planificar estrategias de enseńanza o de aprendizaje. Más bien la función de intercambio de saberes debe tener un carácter colaborativo y simbiótico donde los actores facilitador y estudiante, comparten un mismo interés con la libertad de abordarlo bajo los criterios particulares que el cómo y dónde aplicarlos le proporcionen. Así la construcción del conocimiento será verdaderamente colectiva.

Hemos de encontrar entonces que los actores se convierten a sí mismos como agentes de motivación, reflexión y planificación permanente y profunda para que la satisfacción y el amor por lo que se hace se cultiven exitosamente. La consecuencia inmediata de este interactuar recursivo de actitudes ventajosas es la autorrealización del individuo en virtud de que se siente aceptado por el grupo y como agente participe de las acciones que se realizan, aspecto indispensable en la cohesión e integración del conjunto de individuos en quienes se desarrolla progresivamente características de emprendedor. Se aprecia la capacidad de aceptación de las situaciones vividas lo cual resulta beneficioso para el desarrollo personal y profesional del individuo, ya que asume las experiencias como enseñanzas que permiten una mejor actuación en situaciones similares futuras. El factor emocional surge como abono para que los aprendizajes se internalicen con mayor facilidad y permanencia, propiciando la amistad y el amor entre quienes participan de la educación cooperativa.

Cabe destacar que los egresados que se formen bajo esta filosofía se podrán asociar en organizaciones cooperativa capaces de perdurar en el tiempo con productividad, generando beneficios integrales a sus miembros y a la comunidad a quien sirve. Estos aspectos representan algo que va más allá de una filosofía de trabajo 
Educación cooperativa: una alternativa para generar conocimiento social transformador. cooperativo. Es, y de manera significativa, un modo de vivir y de convivir en armonía con el prójimo, en cooperación para la acción y con el beneficio de un desarrollo socio productivo armónico capaz de lograr la sustentabilidad, la sostenibilidad y en consecuencia un desarrollo verdaderamente endógeno, por lo tanto, la apropiación de una praxis organizacional educativa de autoformación reflexiva, critica, sistémica, recursiva y complementaria en el diseño curricular del área de ingeniería de sistemas, la cual fue objeto de estudio, exhibe características y relaciones señaladas en el Grafico Nro. 2.

\section{Conclusiones}

La consecuencia inmediata de este actuar es la total vinculación que se consolida progresivamente entre el área de conocimiento que aborda y la realidad que se quiere intervenir y transformar. El estudiante debe perder el temor de expresarse en clases o de dirigirse al facilitador, pero para que esto suceda el facilitador debe propiciar un ambiente de cordialidad, fraternidad y amistad, lo cual implica que nuestro pensar y actuar debe cambiar y comprender que nosotros también fuimos y seremos caminantes sobre el sendero del aprendizaje y que podemos aprender cosas magnificas y descubrir maravillas junto con aquellos "maestros" que se sientan en un pupitre a escucharnos narrar algunas experiencias y para el compartir de saberes. La intención subyace en una relación simbiótica, reflexiva y que trasciende más allá de unos contenidos particulares de un programa sinóptico que contribuye en el desarrollo de habilidades: La construcción de competencias para la vida como ser integral y holístico mediante una educación cooperativa.

En igual orden de ideas, el conocimiento es un bien inacabado: el objetivo es problematizar las circunstancias, factores, elementos $\mathrm{y}$ actores para que las soluciones planteadas en un principio puedan recomponerse para adecuarse a la dinámica que emerge reclamando nuevas respuestas. Así pues la función de investigación reclama sus espacios como fuente de nuevo conocimiento que se deriva de una realidad innegable que coexiste en franca lucha con las teorías que rigen los paradigmas agonizantes. Se abre la puerta a la curiosidad, la sospecha y el asombro como elementos enriquecedores de 
la praxis educativa bajo la filosofía cooperativista y de construcción permanente que se imbrican en los intentos exitosos y fallidos de reformas curriculares orientados en algunos casos a modificar la apariencia y en otros, para cambiar la esencia educativa.

Así pues, el proceso de construcción curricular permanecerá en una constante incertidumbre para que la novedad de lo más adecuado pueda surgir según se suceden los cambios en la realidad, por lo que nada es definitivo. Tal vez podríamos llamarlos intentos o simplemente experiencias, donde cada vivencia se construye sobre una experiencia anterior tratando de superar los detalles que afloran y que se enfrentan a nuevas circunstancias, razón que determina la participación de los actores involucrados como condición imprescindible para construir bajo la figura de la resignificación de lo observado, estudiado, aprendido y sentido en relación a los diferentes modelos sociales que alimentan nuestras realidades. Los pilares del conocimiento social transformador se consolidan bajo la cosmovisión integradora de los componentes que hologramáticamente conforman el todo.

Grafico Nro. 3: Integración de los conocimientos construidos desde el colectivo para una praxis educativa cooperativa orientada al desarrollo conocimiento social transformador.

Fuente: Valenzuela, R. (2017).

Indiscutiblemente, la promoción de conocimiento social transformador se logra mediante la interacción dialógica con el otro en una combinación sinérgica de saberes en donde cada parte proporciona ópticas e ideas que permiten dilucidar sobre un tema, necesidad de conocimiento o innovación productiva en pro de alcanzar objetivos, propósitos y metas de corte colectivo. De ahí que el aprendizaje cooperativo propone la armonía entre las y los actores del hecho educativo de tal manera que todos y todas de forma comprometida inicien la búsqueda de respuestas a las exigencias sociales amparadas en un creciente desarrollo tecnológico. Los saberes, el conocimiento y las competencias se desarrollan producto de la ejercitación y práctica del conocimiento que se construye sobre la base de un conocimiento previo. Es decir, es un proceso mental que no ocurre de manera aislada y que es acumulativo con el paso del tiempo, el cual proporciona vivencias en el intercambio de saberes en el desempeño de las diferentes funciones y roles que tiene 
Educación cooperativa: una alternativa para generar conocimiento social transformador.

Gráfico Nro. 2

Tejido de relaciones que caracterizan la praxis organizacional educativa

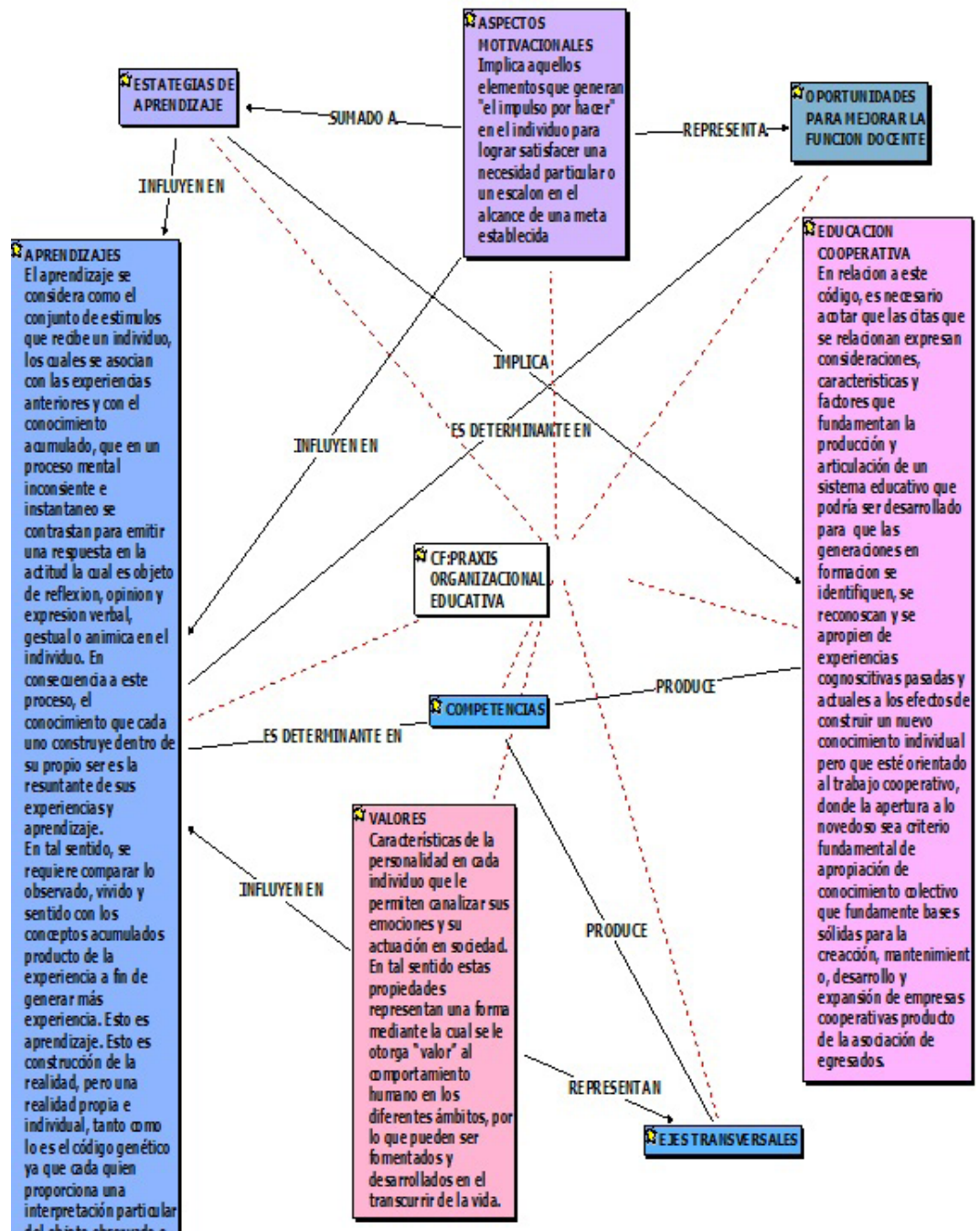

Fuente: elaboración propia (2017) 
el ser humano. Así pues, el ejercicio de la función como facilitador en un espacio que propicia la discusión sobre temas particulares relacionados con un área del saber y estructurado por contenidos programáticos orientados a proporcionar habilidades, destrezas y actitudes entre los estudiantes, implica que los actores partícipes del proceso desarrollen de forma simultánea la reflexión de las vivencias escolarizadas y no escolarizadas dentro del contexto donde se suceden con la ferviente identificación de su significado para lograr una comprensión de las razones, elementos y características que lo representan.

Esta reflexión, implica la generación de ideas particulares en el individuo producto de la apropiación de la vivencia que socializada adecuadamente y con la orientación de aquellos poseedores de un conocimiento más amplio y profundo en el área respectiva, permite el surgimiento de un nuevo saber desde el punto de vista cognoscitivo y operativo, identificados generalmente como la teoría y la práctica. Esta es la verdadera apropiación del conocimiento: cuando los estudiantes reciben una información que luego se vive en el momento en que las circunstancias de la realidad reúnen los elementos para poder aplicarla con las particularidades que el proceso subjetivo que la mente del sujeto le proporciona: cada individuo interpreta

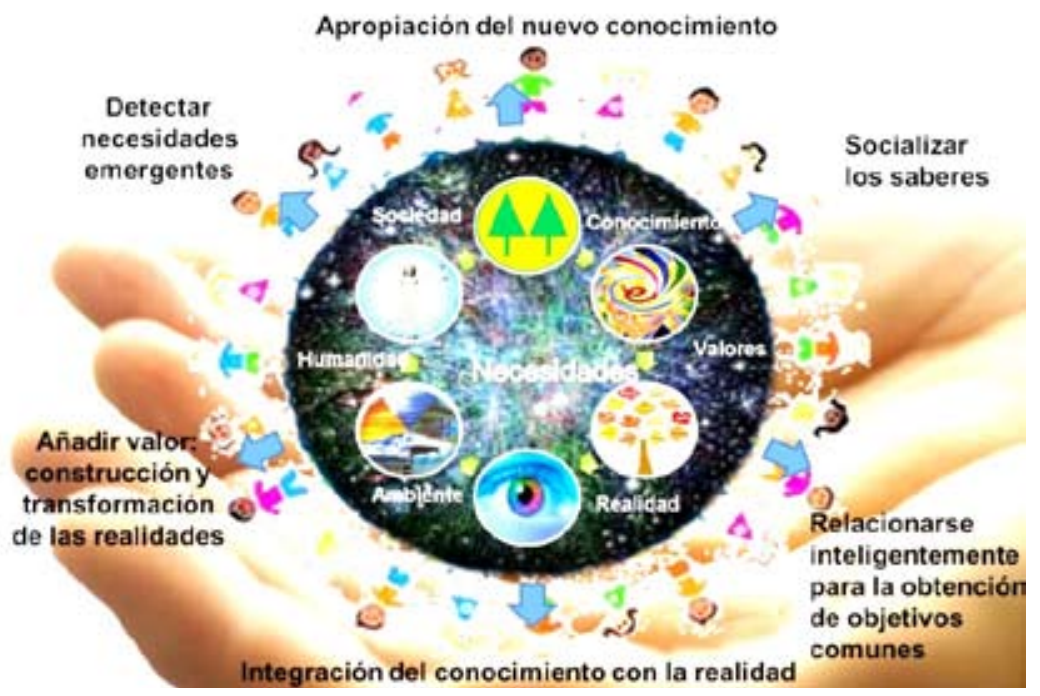

y reflexiona de acuerdo con la suma de conocimientos previos. 
Educación cooperativa: una alternativa para generar conocimiento social transformador.

Ahora bien, la acción humana es la unidad mínima y fundamental de la realidad social y se define como una conducta alineada hacia el logro de fines y metas u otros orientados a mejorar la calidad de vida del individuo. En tal sentido, el aprendizaje cooperativo puede entenderse como una filosofía de vida donde valores y actitudes son los pilares fundamentales para la realización del trabajo en armonía y con desempeño exitoso capaz de desarrollar continuamente competencias para la vida cotidiana. La disposición al trabajo, la alegría, el entusiasmo, el compartir, la constante motivación, la creatividad y la innovación, la amistad, el compańerismo, la solidaridad, el apoyo mutuo, la responsabilidad y la hermandad se convierten en los cimientos que solidifican la estructura del aprendizaje cooperativo y en consecuencia promotor del conocimiento social transformador. Entonces por qué dejar de lado a los estudiantes a la hora de planificar estrategias de enseñanza o de aprendizaje. Más bien, la función de intercambio de saberes debe tener un carácter colaborativo y simbiótico donde los actores facilitador y estudiante, comparten un mismo interés con la libertad de abordarlo bajo los criterios particulares que el cómo y dónde aplicarlos le proporcione. Así la construcción del conocimiento será verdaderamente colectiva.

En éste orden de ideas, la investigación educativa debe impulsar la transformación universitaria en el marco de la territorialización y/o municipalización. Por consiguiente, las universidades deben internalizar y construir un estilo innovador que proporcione libertad a los actores para construir su conocimiento, descubrir nuevos saberes y ofrecer alternativas seguras, válidas, confiables de crecimiento individual y colectivo bajo el esquema de renovación en los diseños curriculares y surgimiento de competencias vanguardistas capaces de sembrar en los partícipes la creatividad y el sentido de conservación planetaria. La educación cooperativa implica formar ciudadanos con capacidad crítica, objetiva, con sentido humano y participativo, con profunda preocupación por su entorno, donde la racionalidad no es símil de números en un estado de ganancias y pérdidas. Por el contrario, es reflejo de su interés por superar los problemas de manera colaborativa donde la cooperación es fundamental para encontrar soluciones integrales a problemas cotidianos con trascendencia global y compleja. 
En todo este escenario de construcción no podemos dejar de lado la función relevante que cumplen las tecnologías de información y comunicación como herramientas que permiten el rompimiento de las fronteras escolarizantes. Por supuesto, el empleo de la educación a distancia posee ventajas insuperables puesto que proporciona un medio directo y constante para la relación entre el facilitador y el alumno, en un ambiente de múltiples recursos didácticos y que se encuentra saturado de información en muchos casos confiables y validados, pero en otros no tanto. Por lo tanto, es el momento en el tiempo que brinda la oportunidad para aprender a emplear tales herramientas con el apoyo de los estudiantes cuya generación es nativa de la tecnología, por lo que se les hace fácil y agradable. Esto es una ventaja significativa que podemos aprovechar al máximo.

Finalmente, y considerando las aristas ontoepistemicas que influyen en la investigación educativa orientada a la construcción del conocimiento social transformador, la educación cooperativa debe ser revisada constante y exhaustivamente a los efectos de detectar las ventajas y oportunidades, creando nuevos proyectos conjuntos que de forma prospectiva puedan adelantarse a los movimientos del entorno. Maslow (1992), afirma que cuando una necesidad es satisfecha, surge en su lugar otra de manera inmediata, vale decir entonces que la naturaleza dinámica del ser humano determina variabilidad e incertidumbre en el direccionamiento de su realidad. Por lo tanto, esta afanosa condición cambiante debe ser detectada prontamente para mantenerse a la vanguardia conocimientos apropiados a las nuevas circunstancias. Por otro lado, la investigación y control de la educación cooperativa requiere la identificación de las debilidades y amenazas que afronta en su cotidianidad a los efectos de establecer oportunidades de mejora y planes de contingencia capaces de subsanar los embates inesperados.

\section{Referencias bibliográficas}

Bordeleau, Y., Brunet, L., Haccoun, R., Rigny, A., \& Savoie, A. (2000). Modelos de investigación para el desarrollo de recursos humanos. México: Trillas.

Colás, M. (1998). Enfoques en la metodología cualitativa: sus prácticas de 
Educación cooperativa: una alternativa para generar conocimiento social transformador.

investigación. En Buendía, L., Colás, M. y Hernández, F. (Comps.). Métodos de investigación en Psicopedagogía (pp. 225-249)

Freire, P. (1970). Pedagogía del oprimido. Montevideo: Tierra Nueva.

González, J. (2008). La mentefactura: factor fundamental para las economías. Recuperado de: http:/www.juridicas.unam.mx/publica/librev/rev/rap/cont/114/pr/pr9.pdf

Hurtado, J. (2000). El Proyecto de investigación. Metodología de la investigación holística. Caracas: Fundación Sypal.

Martínez, M. (2007). La Investigación Cualitativa Etnográfica en Educación. Manual teórico-práctico. México: Trillas.

Martínez, J. (1972): Las Cooperativas de Venezuela. Centro Gumilla, Caracas.

Mas, M. (2005). Desarrollo Endógeno: Cooperación y competencia. Caracas: Panapo

Maslow, A. (1992). Motivación y personalidad. Madrid: Ediciones Díaz de Santos. Recuperado de: http://books.google.co.ve/books?id=8wP dj2Jzqg0C\&printsec=frontcover

McClelland, D. (1989) Estudio de la motivación humana. Madrid: Narcea. Recuperado de: http://books.google.co.ve/books?id=3fKGr602 DTcC\&printsec $=$ frontcover

Owen, R. (1816). A new view of society. Recuperado de: http://www. marxists.org/reference/subject/economics/owen/ch01.htm

Padrón, J. (1994): ¿Qué es teoría? En investigación y docencia. Caracas: Publicaciones del Decanato de Postgrado, USR Recuperado de: http://padron.entretemas.com/ques_teoria.htm

Parsons, T. (1951). The social system. Londres: Routledge \& Kegan Ltd. Recuperado de: http://books.google.co.ve/books?id=t2vkBZylkdQ C\&printsec=frontcover\&source=gbs_v2_summary_r\&cad $=0 \# \mathrm{v}=\mathrm{o}$ nepage $\& \mathrm{q}=\& \mathrm{f}=$ false

Valenzuela, R. (2011): Aproximación teórica hermenéutica de la praxis educativa orientada al desarrollo sustentable. La formación de emprendedores cooperativistas en el contexto de la Universidad Rómulo Gallegos. Tesis Doctoral, UNERG. Guárico -Venezuela.

Villarreal, R. \& Villarreal, T. (2003). IFA. La empresa competitiva sustentable en la era del capital intelectual. México: Mac Graw Hill Interamericana. 